\title{
A Global-Local Contrast based Image Enhancement Technique based on Local Standard Deviation
}

\author{
Archana Singh \\ Ch. Beeri Singh College of Engg \& Management \\ Agra, India
}

\author{
Neeraj Kumar \\ Hindustan College of Science \& Technology \\ Mathura, India
}

\begin{abstract}
Contrast Enhancement plays an important role in digital image processing. It enables the human and automated perceiver to find out the less obvious information in the original image. Global Contrast Enhancement techniques are used in a wide range of applications to enhance the quality of an image. Local contrast enhancement also plays an important role for a detailed visibility of an image. In this paper we tried to enhance the contrast of an image using Global enhancement technique complemented with local image contrast enhancement methods. This paper proposes a method to obtain good contrast image based on its local and global information and by changing the gray values of pixels according to the objective gray scaled image.
\end{abstract}

\section{General Terms}

Image Processing.

\section{Keywords}

Image enhancement, Local contrast enhancement, Global contrast enhancement, local standard deviation.

\section{INTRODUCTION}

In digital image processing Contrast is defined as the difference in brightness at any particular area of image. It is the very first and important process that is used to improve the quality of an image for both human and computer vision. It works to increase the dynamic range of input gray level of an image. Good quality images or pictures are well suitable with consumer electronic products like television, robotics etc. However, the images that are captured by camera or other optical system may have low contrast. There are several reasons of such type of low contrast image: the poor quality of the used capturing device, lack of knowledge of the operator or other external conditions at the time of acquisition [13]. To enhance the contrast of low contrast image type, various contrast enhancement techniques can be applied in spatial and/or frequency domains [16].

One of the promising techniques for image contrast enhancement is Global Contrast Enhancement (GCE). These methods works fast in terms of processing speed as compared to the local contrast enhancement [17]. Global enhancement methods enhance an image globally. The most common methods used for global contrast enhancement are: Linear Contrast Enhancement (LCE), Histogram Equalization (HE) etc. In Linear contrast enhancement, end points of the gray scale distribution are pulled to the end points of palette [18]

In the palette lowest color is used to display minimum gray value of the image. All the values are redistributed using Rest Palette Color [2]. Another well known method for global contrast enhancement [4] is histogram equalization. It is the most commonly applied method due to its simplicity, effectiveness and well performance for most types of images. Histogram equalization redistributes the gray levels of the image according to the probability distribution of the input gray levels [5]. These methods can be broadly classified in two categories - global and local histogram equalization [6]. Global histogram equalization (GHE) method enhances the image globally by using the histogram information of the entire input image for its transformation function [4]. This method is unable to enhance an input image using its local contrast features as gray levels having very high frequency rule over those gray levels having very low frequency. Usual methods of contrast enhancement work globally with the image and do not pay special attention to smaller regions of low contrast [10]. Such images require local treatment to enhance the brightness of smaller regions as shown in figures. Fig. 1(a) is the original image and Fig. 1(b) is the enhanced image based on global contrast enhancement.

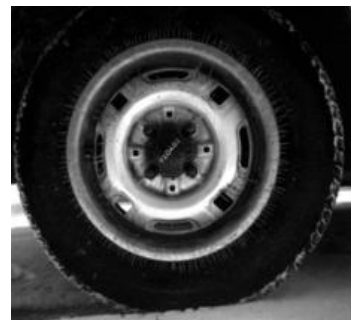

Fig. 1(a)

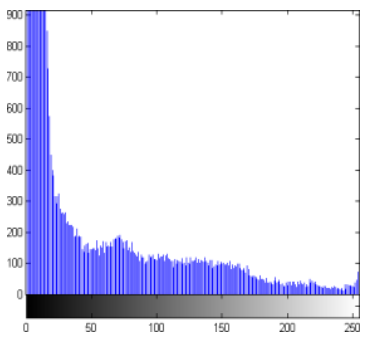

Histogram of $1($ a)

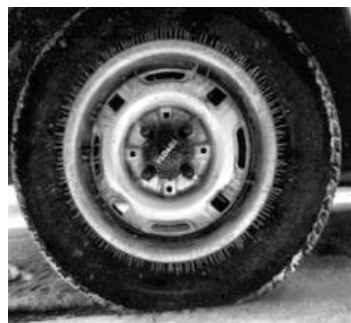

Fig. 1(b)

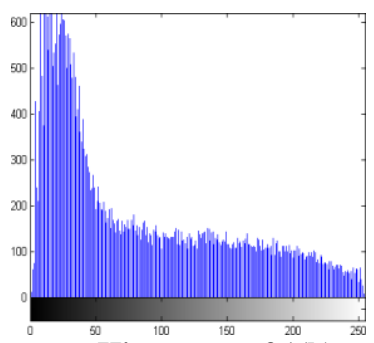

Histogram of $1(b)$
Local Histogram Equalization (LHE) is the extension of this histogram equalization that uses the transformation function of histogram equalization for the blocks [18]. It modifies the centre pixel of block in order to achieve contrast as shown in fig. 1(a) and 1(b). It uses local standard deviation (LSD) and local mean to enhance an image [1]. 


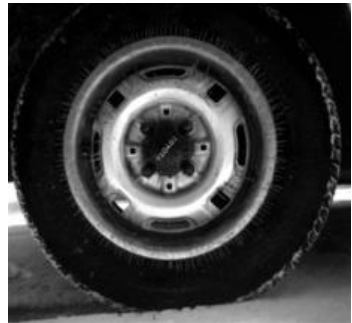

Fig. 2(a)

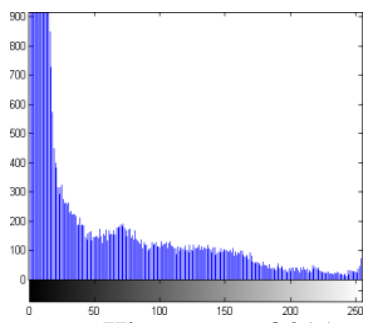

Histogram of 2(a)

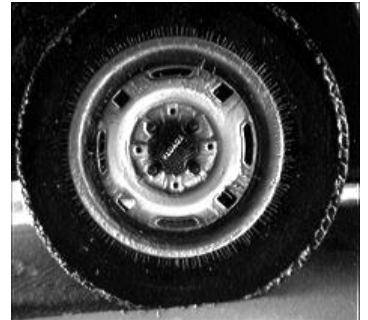

Fig. 2(b)

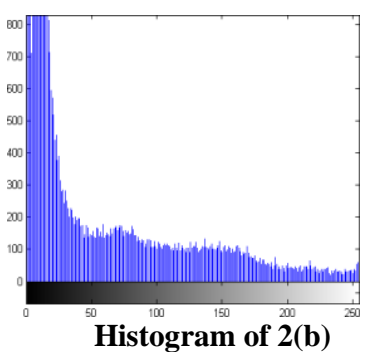

Histogram of 2(b)
Dorst [3] adapted histogram stretching method over a neighbourhood around the candidate pixel for local contrast stretching. A numerous modifications [9, 12] of histogram equalization are introduced based on adapting the same over a sub region of the image. Narendra and Fitch [11] introduced an amplification factor too, a function of the pixel based on the local gray level statistics over the same neighbourhood in that contrast gain is inversely proportional to local standard deviation [LSD]. Lee [7,8] introduced a method that was based on the use of local statistics of a predefined neighbourhood in modifying the gray value of a pixel.

Zheng and Wang [19] proposed a piecewise linear function model based on local standard deviation to increase the luminance at every local region present in the image. In this model integral image is used to fast compute the mean and local standard deviation in all local region centred at each pixel. Dah-Chang [2] designed a method for image enhancement with contrast gain that is constant and inversely proportional to the LSD produces either ringing artifacts or noise over enhancement due to the use of too large contrast gains in regions with high and low activities and proposed a new method in that contrast gain is a non-linear function of LSD.

\section{RELATED WORKS BASED ON LOCAL STADARD DEVIATION}

There are various methods that are used for contrast enhancement based on local standard as discussed below:

Narendra and fitch [11] proposed a method for contrast enhancement using local standard deviation as discussed:

$f\left(i_{i}, j\right)=m_{x}\left(i_{i}, j\right)+\frac{D}{\sigma_{x}(i, j)}\left[x\left(i_{i} j\right)-m_{x}\left(i_{i}, j\right)\right]$

Here $f\left(i_{i}, \hat{j}\right)$ is the gray scale value of enhanced image. $x\left(i_{i} \hat{j}\right)$ is the value of pixel in original image. D is a constant. $m_{x}\left(i_{v} j\right)$ is local mean and $\sigma_{x}\left(\hat{i}_{x} j\right)$ is the local standard deviation.

Dah-Chang [2] proposed another method based on LSD information. This method was proposed for medical images as discussed here:

$f\left(i_{x}, \hat{j}\right)=m_{x}\left(i_{x}, \hat{j}\right)+k\left(i_{i}, \hat{j}\right)\left[\frac{x(i, j)-m_{y}(i, j)}{\sigma_{x}(i, j)}\right]$

Here $k\left(\hat{i}_{w} \hat{j}\right)$ is known as contrast gain.
Another method Multi-Scale Adaptive Gain Control was discussed by Schutte [15] to enhance an image using LSD. This method uses both global and local information of the input image given as:

$O=i+\sum_{j=1}^{k} M \frac{C_{f}}{L S D_{f}}\left(i-m_{j}\right)$

Here $\mathbf{O}$ is the pixel value of output image.

$\mathbf{i}$ is the pixel value of original image. $\mathbf{M}$ is the global mean of the input image, $C_{j}$ is the gain factor that controls the enhancement level per window, LSD is the local standard deviation per window and $\mathbf{m}$ is the local mean of the window.

\section{GLOBAL LOCAL CONTRAST ENHANCEMENT FOR LOCAL REGION}

Global contrast enhancement or local contrast enhancement method are not be suitable for those images that are poor in global contrast as well as in local contrast. These types of images require a method in that global contrast enhancement and local contrast enhancement are applied.

This proposed work is based on the combination of global contrast enhancement and local contrast enhancement for such type of information that is existing in less dynamic region of image. This is done by subtracting two subsequent gray scale values of the image.

$0=I+\frac{M * C_{\mathrm{f}}}{\sigma_{\mathrm{f}}}\left[m_{\mathrm{i}}-m_{\mathrm{i}+1}\right]$

where $\mathbf{O}$ is the pixel's gray level value of enhanced image corresponding to I pixel's gray value of original image. $\mathbf{M}$ is the global contrast gain control with the range of $[0,1.5] . C_{\tilde{t}}$ is the local contrast control with the range of $[0,1] . \sigma_{i}$ is the local standard deviation of the window. $m_{i}$ is the pixel's gray scale value present in the original image and $m_{i+1}$ is the subsequent pixel's gray scale value of the original image. This method slides pixel by pixel into the entire image.

\section{EXPERIMENTAL RESULT}

In order to evaluate the results of our proposed method, we have done subjective as well as objective evaluation.

\subsection{Subjective Evaluation}

Subjective evaluation is done by human being. This is necessary to find out the quality of generated enhanced image.

Table 1. Subjective results of contrast evaluation

\begin{tabular}{|l|l|l|l|l|l|l|l|l|l|}
\hline Method & \multicolumn{2}{|l|}{$\begin{array}{l}\text { Original } \\
\text { Image }\end{array}$} & \multicolumn{3}{l|}{$\begin{array}{l}\text { Based on } \\
\text { equation no. }\end{array}$} & \multicolumn{3}{l|}{$\begin{array}{l}\text { Proposed } \\
\text { work }\end{array}$} \\
\hline Rank & $\mathbf{1}$ & $\mathbf{2}$ & $\mathbf{3}$ & $\mathbf{1}$ & $\mathbf{2}$ & $\mathbf{3}$ & $\mathbf{1}$ & $\mathbf{2}$ & $\mathbf{3}$ \\
\hline cameraman & 2 & 1 & 3 & 1 & 3 & 2 & 3 & 2 & 1 \\
\hline Eight & 3 & 1 & 2 & 3 & 3 & 0 & 6 & 0 & 0 \\
\hline Tire & 1 & 3 & 2 & 4 & 0 & 2 & 5 & 0 & 1 \\
\hline Pout & 4 & 2 & 0 & 1 & 3 & 2 & 1 & 2 & 3 \\
\hline Totals & 10 & 7 & 7 & 9 & 9 & 6 & 15 & 4 & 9 \\
Rank & & & & & & & & & \\
\hline
\end{tabular}




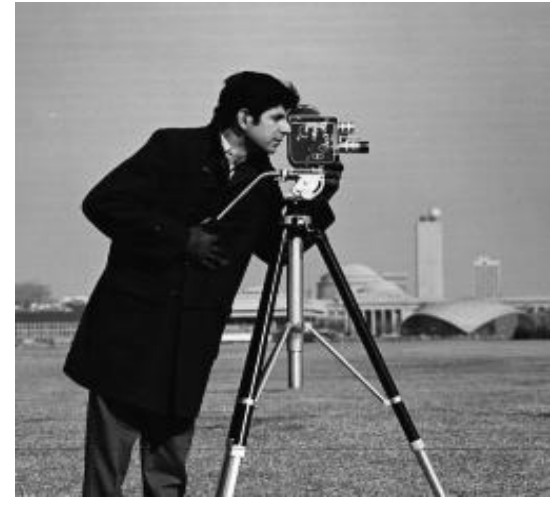

(a) Cameraman

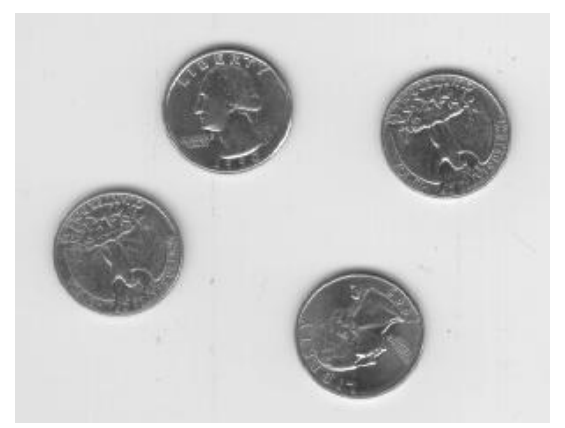

(a) Eight

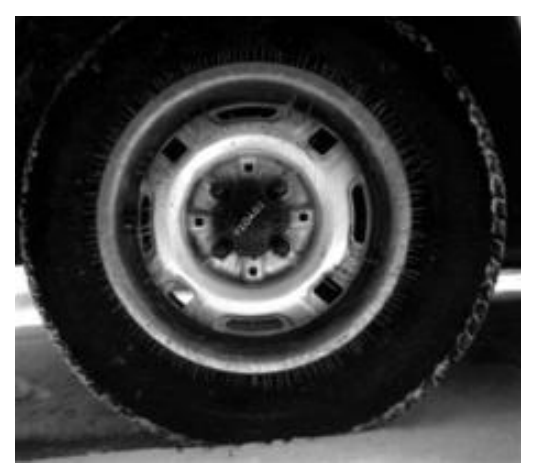

(a) Tire

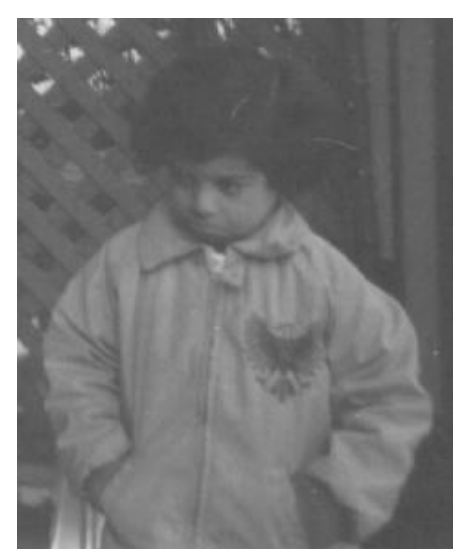

(a) Pout

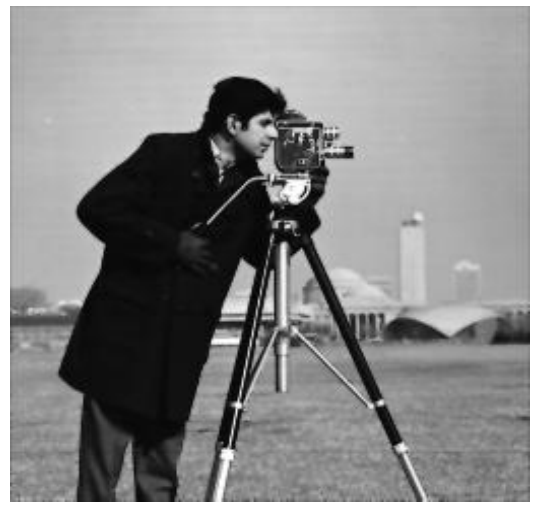

(b) Cameraman

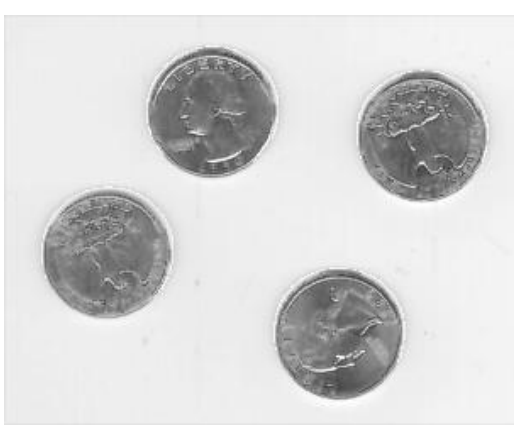

(b) Eight

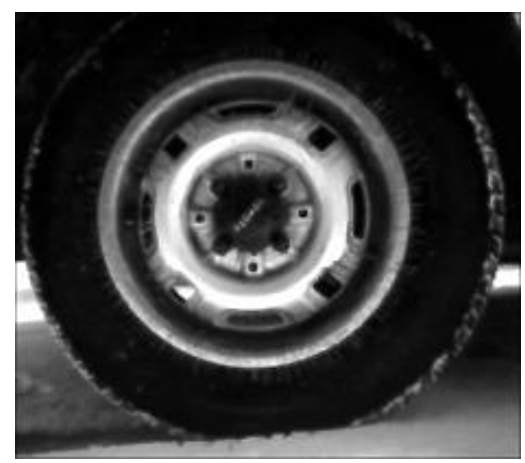

(b) Tire

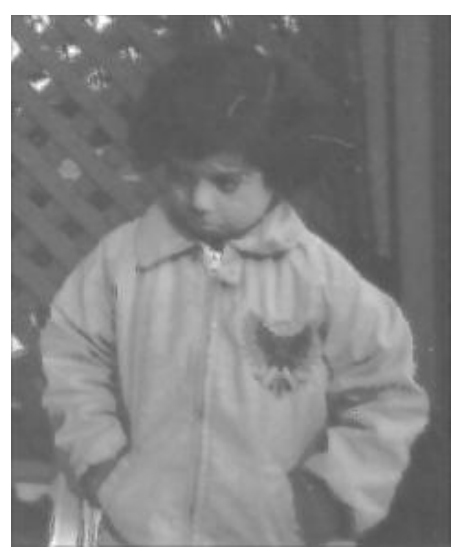

(b) Pout

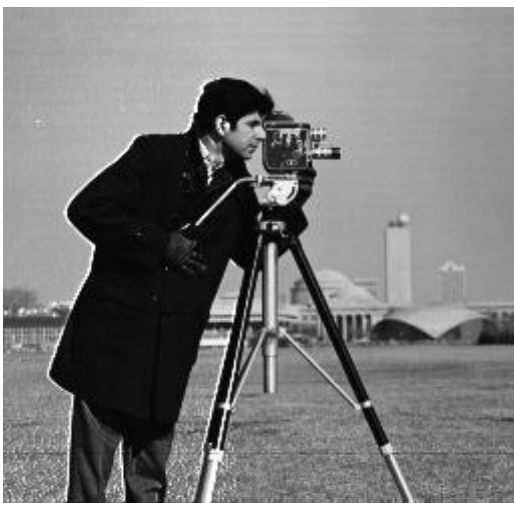

(c) Cameraman

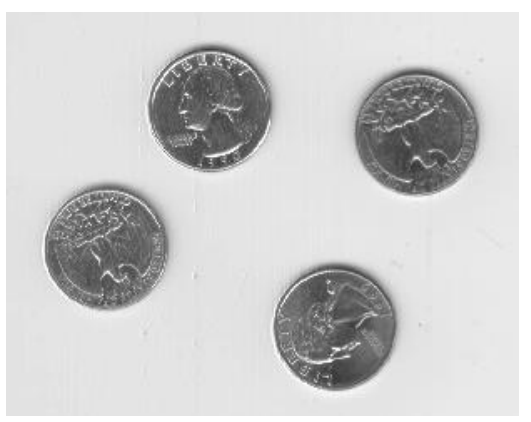

(c) Eight

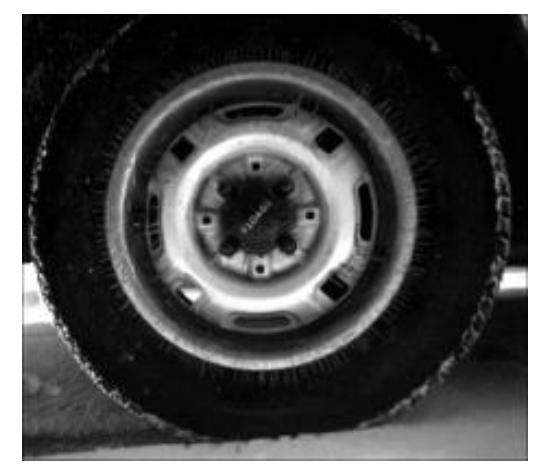

(c)Tire

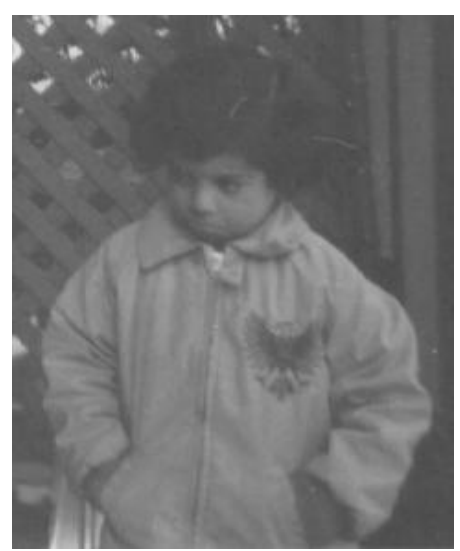

(c) Pout

Fig. 3. Enhancement results: (a) - Original Image, (b) based on equation no.-3, (c) based on proposed method 
This evaluation has been done by 6 interpreters. They gave their score from 1 to 3 to each image, where 1 is the best and 3 is the worst score. Table I shows the results of subjective evaluation. Image having score 1 contains more brightness as compare to other two other images and image having score 3 shows that the image has very low brightness as compare to others.

The results of Table I. and enhancement results of Fig. 3 are going in the favour of our proposed method. In this table total rank of proposed work is showing that the images are enhanced using this method has been scored 15 times best as compare to others

\subsection{Objective Evaluation}

For the objective evaluation detail variance (DV) and background variance (BV) of images are calculated. These detail variance and background variance are calculated using the method described by Ramponi, Strobel, Mitra and. Yu, [14].

According to this method the values of detail variance and background variance are obtained as following: first the variance of the gray levels in the neighbouring pixels is calculated at each pixel in the image. Next the pixel is classified to the foreground when the variance of the gray level is more than a threshold and the pixel is classified to the background when the variance of the gray level is lower than a threshold. The averaged variance of all pixels included in the foreground class is DV and the averaged variance of all pixels included in the background class is BV. When the value of DV of the resulted image increases and the value of $\mathrm{BV}$ is not changed compared to the original gray image. Then it is supposed that efficient contrast enhancement has been achieved by Ramponi [14]. Table IV shows the detail variance and background variance in images enhanced by equation no. 3 and proposed work.

By analyzing the following results it has been observer that the proposed work produces better image with natural enhancement as compare to the existing work.

Table 2. Objective results of contrast evaluation

\begin{tabular}{|l|l|l|l|l|l|l|}
\hline Image & \multicolumn{2}{|l|}{ Original } & \multicolumn{2}{l|}{$\begin{array}{l}\text { Existing } \\
\text { work }\end{array}$} & \multicolumn{2}{l|}{$\begin{array}{l}\text { Proposed } \\
\text { work }\end{array}$} \\
\hline Rank & DV & BV & DV & BV & DV & BV \\
\hline a) cameraman & 2.7 & 2 & 3.1 & 2.2 & 3.5 & 2.2 \\
\hline b) eight & 5.5 & 1 & 4.8 & 1 & 3.7 & 1 \\
\hline c) tire & 3.3 & 0.6 & 3.5 & 1.1 & 3.9 & 1.1 \\
\hline d) pout & 2.7 & 2.6 & 3.3 & 2.7 & 3.9 & 2.7 \\
\hline
\end{tabular}

\section{CONCLUSION}

The basic idea behind the proposed work is to enhance an image using local standard deviation. This proposed method for contrast enhancement is based on global contrast enhancement and local contrast enhancement. This method includes the local and global features of the given image to find out the information that is present in the minute area of image.

\section{REFERENCES}

[1] Archana, Verma, Akhilesh, Goel, Dr. Savita and Kumar, Neeraj. 2013. Gray Level Enhancement to Emphasize Less Dynamic Region within Image Using Genetic Algorithm. In $3^{\text {rd }}$ IEEE International Advances computing Conference IACC-2013, ISBN: 987-1-46734528-6.

[2] Chang, D.C. and Wu, W.R. 1988. Image contrast enhancement based on a histogram transformation of local standard deviation. In IEEE Trans. MI, Vol. 17. no. 4, pp. 518-531.

[3] Dorst, L. 1982. A local contrast enhancement filter. In Proc. 6th Int. Conference on Pattern recognition. pages 604-606. Munich. Germany.

[4] Gonzales, R. and Winter, P. 1978. Digital Image Processing. Addison Wesley.

[5] Jain, K. 1997. Fundamentals of digital image processing. Englewood Cliffs. NJ. Prentice-Hall.

[6] Kim, J.Y., Kim, L.S., and Hwang, S.H. 2001. An advanced contrast enhancement using partially overlapped sub-block histogram equalization. In IEEE Transactions on Circuits and Systems for Video Technology. Vol.11. pp. $475-484$.

[7] Lee, J.S. 1980. Digital image enhancement and noise filtering by use of local statistics. In IEEE Trans. on Pattern Analysis and Machine Intelligence. PAMI-2:165.

[8] Lee, J.S. 1981. Refined filtering of image noise using local statistics. Computer Graphics and Image Processing. 15:380.

[9] Mukherjee, D. and Chatterji, B.N. 1995. Adaptive neighborhood extended contrast enhancement and its modification. Graphical Models and Image Processing. 57:254-265.

[10] Mukhopadhyay, S. and Chanda, B. 2000. Local Contrast Enhancement of Grayscale Images using Multiscale Morphology.

[11] Narendra, P.M. and Fitch, R.C. 1981. Real-time adaptive contrast enhancement," IEEE Trans. PAMI, Vol.3, no. 6, pp.655-661.

[12] Pizer, S.M., Amburn, E.P., Austin, J.D., Cromartie, R., Geselowitz, A., Geer, T., Romeny, B.H., Zimmerman, J.B. and Zuiderveld, K. 1987. Adaptive histogram modification and its variation. Computer Vision. Graphics and Image Processing, 39:355-368.

[13] Rajamani, V., Babu, P. and Jaiganesh, S. "A Review of various Global Contrast Enhancement Technique for still Images using Histogram Modification Frame work". International Journal of Engineering Trends and Technology. April 201. Vol. 41. Issue 4.

[14] Ramponi, G., Strobel, N., Mitra, S.K. and Yu, T.-H. 1996. "Nonlinear Unsharp Masking Methods for Image Contrast Enhancement". Journal of Electronic Imaging, Vol. 5(3), pp. 353- 366.

[15] Schutte, K. 1997. Multi-Scale Adaptive Gain Control of IR Images. Infrared Technology and Applications XXIII. In Proceedings of SPIE Vol. 3061, pp.906-914. 
[16] Singh, S.Somorjeet, Singh, Th. Tangkeshwar, Devi, H. Mamata and Sinam,Tejmani. 2012. "Local Contrast Enhancement Using Local Standard Deviation," International Journal of Computer Applications (0975888). Vol. 47- No.15.

[17] Singh, S.Somorjeet, Singh, Th. Tangkeshwar, Gourakishwar, N. and Devi, H. Mamata. 2012. "GlobalLocal Contrast Enhancement". International Journal of Computer Applications (0975-888). Vol. 54- No.10.
[18] Verma Akhilesh and Archana. 2012. "A Survey on Image Contrast Enhancement Using Genetic Algorithm". International Journal of Scientific and Research Publications. Vol.2. Issue 7. ISSN 2250-315.

[19] Zheng, Danian, Wang, Jiaxin and Xiao, Zhenghong. 2005. "Image Enhancement Based on Local Standard Deviation". Journal of Information \& Computational Science 2: 2. 429-437. 